tique, des données qui, quoique n'étant pas vraies en manière absolue, sont toutefois bien comparables entre elles.

(Istituto di Merceologia della Università di Roma, Ottobre 1948.)

\title{
DOSAGE COLORIMÉTRIQUE DE LA CASÉINE, DE LA LACTALBUMINE ET DES POLYPEPTIDES DU LAIT
}

\author{
par
}

\author{
ETIENNE GOIFFON \\ Ingénieur I. A. N.
}

Dans un mémoire précédent, nous avons indiqué une méthode colorimétrique de dosage des protides du lait. En présence de la tyrosine et du tryptophane contenus dans ces protides, on obtient une réduction proportionnelle du réactif phospho-tungstomolybdique (réactif des phénols). On mesure la coloration bleue formée au colorimètre ou au photomètre.

Nous avons indiqué dans cette publication tous les détails de technique nécessaires. Il était intéressant de mesurer les protides en bloc, car c'est jusqu'à présent ce qui a été proposé le plus souvent dans les manuels au moyen du dosage de l'azote total : c'est cette donnée, d'ailleurs, qui fournit la valeur alimentaire du lait du point de vue substances azotées, dont la valeur nutritive est sensiblement la même, qu'il s'agisse de caséine, d'albumine ou de protides. Il n'en: est pas moins vrai qu'il peut être utile de doser séparément chacun de ces trois éléments.

Nous nous proposons de donner ici un procédé basé sur le même principe colorimétrique nous permettant d'isoler dans une même suite d'opérations, chacun de ces trois protides et de les doser en mesurant leur pouvoir chromogène, dû à leur teneur en tyrosine et en tryptophane, vis-à-vis du réactif des phénols.

\section{Intérêt du dosage séparé des protides}

\section{a) Caséine.}

Il est inutile d'insister sur l'utilité de mesurer la caséine seule, ce qui est d'un intérêt bien connu soit dans l'industrie de la caséine soit dans la fromagerie.

\section{b) Lactalbumine.}

On s'est préoccupé, à eôté de la caséine, de doser la lactalbumine du lait ; cette substance existe à une concentration avoisinant 2 grammes par litre dans le lait de vache, se retrouve dans le petitlait issu de la fabrication des fromages. 
La présence de la lactalbumine dans le lait est un indice que le lait n'a pas été chauffé au delà de la température de précipitation de l'albumine. La lactalbumine représente une partie des albumines du sérum, comme elle, elle est composée d'albumines et de globulines.

Au point de vue pathologique, la lactalbumine augmente dans les infections de la mamelle.

D'autre part, au point de vue industriel, la quantité de lactalbumine contenue dans un lactosérum constitue un aliment azoté de valeur quand on utilise cet aliment pour les animaux.

Enfin, dans la préparation du lactose, la lactalmumine est isolée et sert de base à des préparations industrielles, soit comme aliment azoté, soit comme matière première de transformations chimiques.

Ces raisons sont suffisantes pour que le dosage de la lactalbumine prenne place à eôté de celui de la caséine du lait.

Notons ici que nous appelons albumines les protides qui ne sont pas précipités par l'acide acétique et qui sont précipités par l'acide trichloracétique.

En dehors des albumines et des globulines, il est possible qu'une partie de la caséine légèrement dégradée soit dans un état tel que l'acide acétique ne la précipite plus et que l'acide trichloracétique la précipite encore.

\section{c) Poly peptides.}

La lactalbumine n'est pas le seul corps azoté présent dans le lactosérum ; à côté de ces protides à poids moléculaires élevés, - en général de 70.000 et plus, - le lactosérum contient des polypeptides, c'est-à-dire des protides dégradés, à poids moléculaire beaucoup plus bas.

L'abondance relative de ces polypeptides, est assez eurieuse. Ils peuvent avoir plusieurs sources possibles.

1. Ils proviendraient directement des polypeptides du sérum. On sait que le sang contient une certaine abondance de polypeptides, e'est-à-dire de ces substances précipitables par l'acide phosphotungstique et non précipitables par l'acide trichloracétique. Cependant, dosé par le même procédé, ils représentent 30 milligrammes de tyrosine par litre de sérum, alors que dans le lait, cet indice de tyrosine est de 100 à 200 milligrammes. Y aurait-il un enrichissement de ces substances au passage de la glande mammaire $s$ ans autre transformation ?

2. Ils peuvent être dus à une action protéasique sur la caséine même avec scission de la grosse molécule de caséine jusqu'au stade 
non précipitable par l'acide trichloracétique. Dans ce cas, peu abondants au moment de la traite, ils augmenteraient progressivement avec le temps, au moins jusqu'au moment où la pasteurisation arrêterait l'action diastasique. Nous n'avons pas constaté cette progression ni l'augmentation des polypeptides, après passage du lait cru à l'étuve, ce qui aurait eu lieu si des protéases avaient continué leur action sur un substratum aussi fragile que la caséine. - 3. Enfin, on pourrait envisager, d'une façon bien vague d'ailleurs, le passage dans le lait des polypeptides à la faveur du remaniement que fait subir la glande mammaire aux protides du sang pour les transformer en caséine.

Enfin, à côté de ces polypeptides, il existe dans le lactosérum des groupements aminés simples, et des acides aminés libres, qui ne sont pas précipitables par l'acide phosphotungstique. Nous avions pensé mesurer par le même procédé la tyrosine et le tryptophane libres existant éventuellement dans cette portion.

Mais, nous avons constaté qu'il existe, à côté de ces corps, des substances tels que l'acide urique et des phénols qui réduisent également le réactif des phénols, ce qui nous interdirait tout dosage des acides aminés par voie colorimétrique.

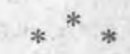

L'uniformité du principe de nos dosages nous incite à décrire presque simultanément leurs techniques.

\section{Méthode générale}

\section{Caséine seule.}

La caséine seule est mesurée par différence entre l'index tyro. sine colorimétrique obtenu avec les protides totaux du lait (d'après la teohnique indiquée dans notre précédent travail) et l'index tyrosine déterminé sur la même quantité de lait dont on a extrait la caséine.

On fera donc deux dosages séparés, l'un des protides totaux du lait, l'autre des protides totaux du lactosérum, la différence étant attribuable à la caséine.

\section{Protides du lactosérum : lactalbumine-polypeptides.}

Dans le lactosérum préparé par précipitation acétique dans l'opération précédente pour le dosage de la caséine, on précipite la lactalbumine par l'acide trichloracétique. Sur le précipité isolé et redissout, on mesure la coloration obtenue par la réaction des phénols.

Dans le liquide débarrassé du précipité de la lactalbumine, on 
précipite par adjonctions d'acide phospho-tungstique, les poly: peptides. Ce précipité isolé et redissout, est soumis à la réaction des phénols et dosé colorimétriquement.

\section{Techniques particulières. - Préparation du lactosérum.}

A $10 \mathrm{~cm}^{3}$ de lait, bien mélangé, on ajoute $15 \mathrm{~cm}^{3}$ d'eau et $2 \mathrm{~cm}^{3}$ d'une solution saturée d'oxalate de soude, dans une éprouvette graduée. Cette adjonction d'oxalate de soude a pour but de rendre insoluble le calcium du lait, qui, sans cela, serait solubilisé par la défécation acide de la caséine et formerait, dans les opérations ultérieures, en milieu alcalin, des précipitations de phosphate de chaux gênantes pour les mesures optiques.

On ajoute alors par affusion successive de l'acide acétique à $5 \%$, jusqu'à ce qu'une floculation brutale, avec une rétraction immédiate des caillots, se produise. On complète immédiatement à $30 \mathrm{~cm}^{3}$ et, après avoir agité, on laisse la coagulation se parfaire pendant 5 minutes.

On filtre sur filtre assez serré, en refiltrant la première partie souvent trouble, jusqu'à ce qu'on obtienne un liquide clair. Ce premier point de l'opération est peut être le plus délicat. Il faut un certain tour de main pour apprécier la quantité d'acide acétique juste nécessaire pour la précipitation de la caséine, qu'un léger excès remettrait en solution.

C'est dans ce liquide contenant le lactose et les sels que se trouvent la lactalbumine, les polypeptides et les acides aminés.

\section{Dosage de la caséine seule.}

A $6 \mathrm{~cm}^{3}$ du filtrat (correspondant à $2 \mathrm{~cm}^{3}$ de lait) on ajoute dans une éprouvette de $50 \mathrm{~cm}^{3}$, de l'eau jusqu'à $25 \mathrm{~cm}^{3}, 1 \mathrm{~cm}^{3}$ de lessive de soude à $36^{\circ} \mathrm{B}$, on mélange et on projette rapidement $3 \mathrm{~cm}^{3}$ du réactif de Folin et Ciocalteu, puis on complète avec de l'eau distillée à $50 \mathrm{~cm}^{3}$. La couleur bleue qui se développe est mesurée au photomètre ou au colorimètre au bout de 15 minutes.

Cette mesure se fait par rapport à une solution de tyrosine à 200 grammes pour 1 litre d'HCL N/10 (1).

D'après Folin et Crocalteu, le nombre de milligrammes de tyrosine contenu dans le liquide albumineux est à multiplier par le coefficient 16,3 pour obtenir ce chiffre en grammes d'albumine.

On prend $2 \mathrm{~cm}^{3}$ de cette solution de tyrosine qu'on traite de la même façon par le réactif des phénols avec un volume total de $30 \mathrm{~cm}^{3}$; on place ces deux solutions colorées dans les godets du colori-

(1) Nous avons préféré un étalon tyrosine à l'étalon résorcine que nous avions proposé dans notre travail précédent, le titre de la solution diluée de résorcine s'alté. rant trop rapidement avec le temps. 
mètre de Duboseq et on ajuste les hauteurs jusqu'à l'égalité de teinte, soit $\mathrm{H}$ la hauteur de la solution de tyrosine, $\mathrm{H}^{\prime}$ la hauteur de la solution de protides, on a $\frac{\dot{H}}{\bar{H}} \times 0,200 \times 16,3=$ quantité de protides.

On peut arriver au même résultat, en comparant, à l'aide du photomètre, les densités optiques obtenues, avec celle d'une courbe de concentration progressive de tyrosine. Le poids de tyrosine trouvé correspondant à $2 \mathrm{~cm}^{3}$ de lait sera divisé par $2 \times 1.000 \times 16,3$ pour obtenir la valeur colorimétrique du lactosérum d'un litre de lait, exprimé en caséine.

Nous aurons ainsi exprimé en caséine, toutes les substances chromogènes autres que la caséine, contenues dans le lait (lactalbumine, polypeptides, tyrosine et tryptophane libres, acide urique, phénols, etc.).

Dans un premier temps, on aura mesuré les protides totaux du lait par le procédé indiqué dans notre premier travail. On soustrait de la concentration trouvée, celle que l'on a déterminée dans le lactosérum. La différence représente la concentration de la caséine seule. Par exemple si le dosage des protides totaux du lait donne 32 grammes, si le pouvoir chromogène du lactosérum, exprimé en caséine, donne 5 grammes, le lait contiendra $32-5=27$ grammes de caséine seule.

\section{Dosage séparé de la lactalbumine.}

S'il y a intérêt à connaître la quantité de lactalbumine, sans les polypeptides, il est aisé d'en faire un dosage séparé. A $15 \mathrm{~cm}^{3}$ du filtrat acétique, obtenu comme précédemment, on ajoute $3 \mathrm{~cm}^{3}$ d'acide trichloracétique à $20 \%$; il se produit un trouble dont on attend la floculation - quand elle s'est produite, on centrifuge, on décante le liquide, généralement opalescent, et on le garde pour le dosage des polypeptides.

Le culot est dissout par quelques gouttes de carbonate de soude à $20 \%$ et de l'eau pour obtenir sa dissolution. On transvase ce liquide dans une éprouvette de $30 \mathrm{~cm}^{3}$ et on amène à un volume de $10 \mathrm{~cm}^{3}$ avec de l'eau distillée la solution albumineuse. On prélève $4 \mathrm{~cm}^{3} \mathrm{du}$ mélange correspondant à $2 \mathrm{~cm}^{3}$ de lait qu'on place dans une éprouvette de $50 \mathrm{~cm}^{3}$. On ajoute de l'eau jusqu'à $25 \mathrm{~cm}^{3}, 1 \mathrm{~cm}^{3}$ de lessive de soude à $36^{\circ} \mathrm{B}$, on mélange et on projette rapidement $3 \mathrm{~cm}^{3} \mathrm{du}$ réactif de Folin et Ciocalteu, puis on complète avec de l'eau distillée à $50 \mathrm{~cm}^{3}$. La couleur bleue qui se développe est mesurée au photomètre ou au colorimètre au bout de 15 minutes. 


\section{Dosage séparé des polypeptides.}

1. Sur le liquide surnageant le précipité à l'acide trichloracétique que nous avons mis de eôté, on ajoute $1 \mathrm{~cm}^{3}$ de réactif de Folin (1). On centrifuge ; on reprend le culot par $10 \mathrm{~cm}^{3}$ d'eau alcaline et on prélève $6 \mathrm{~cm}^{3}$ (correspondant à $3 \mathrm{~cm}^{3}$ de lait).

La suite des opérations et le calcul se font comme dans le cas précédent. En tenant compte des $3 \mathrm{~cm}^{3}$ de prise d'essai.

2. Si on s'intéresse uniquement à l'abondance des polypeptides, on peut abréger le processus. Il suffit de prendre $5 \mathrm{~cm}^{3}$ de lait ; on ajoute $7 \mathrm{~cm}^{3}$ d'eau et $3 \mathrm{~cm}^{3}$ d'acide trichloracétique. On filtre $10 \mathrm{~cm}^{3}$, le liquide doit être parfaitement clair ; il ne contient que les

RÉSULTATS DE QUELQUES DOSAGES

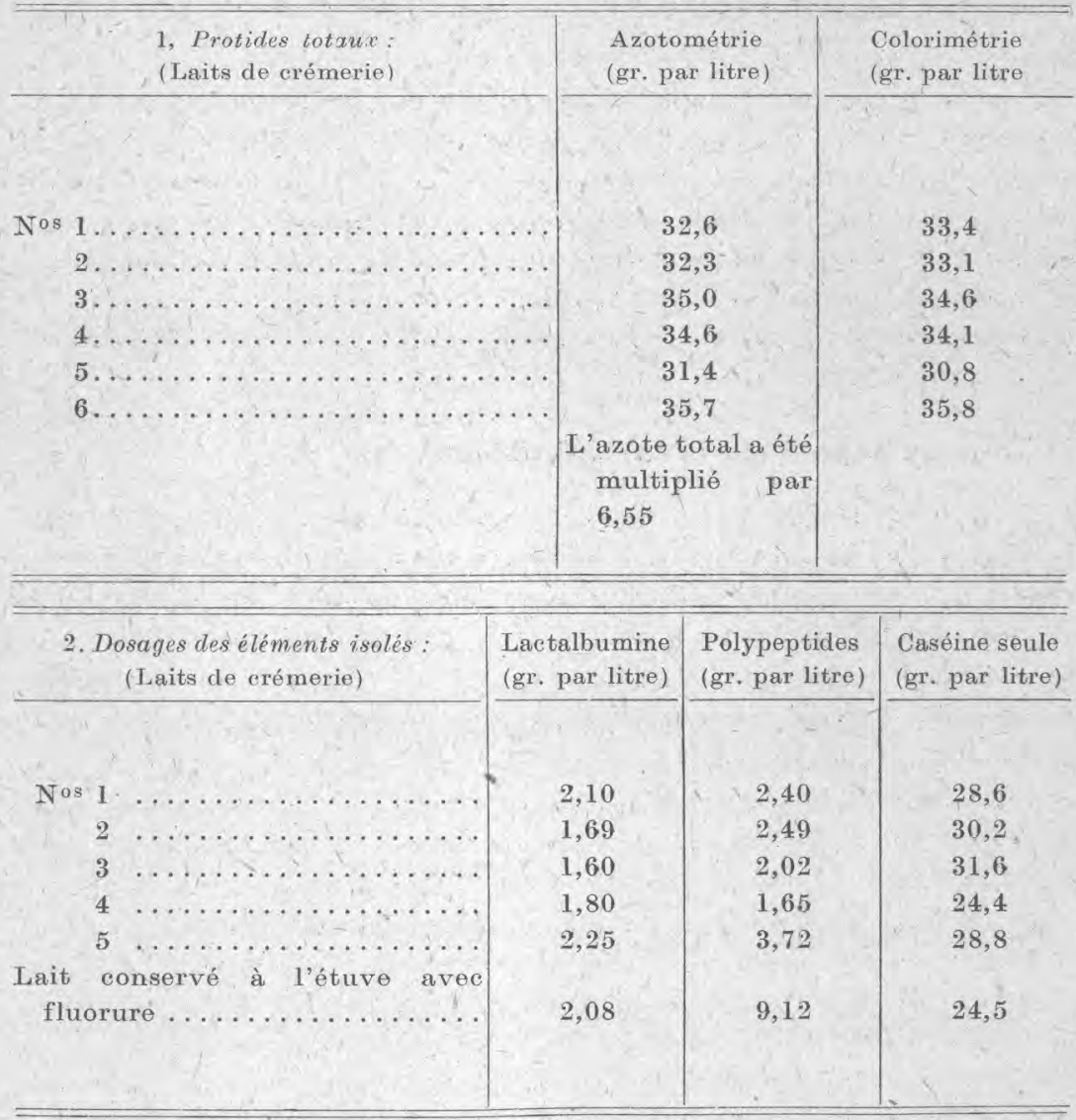

(1) Ce réactif de Folin est la solution d'acide phosphotungstique préparée en vue du dosage colorimétrique de l'acide urique. On l'appelle également « réactif urique». 
polypeptides et les acides aminés. On précipite par $1 \mathrm{~cm}^{3}$ d'acide phosphotungstique (réactif de Folin), et on centrifuge le précipité. On reprend le culot, comme dans l'opération précédente ; on le dilue à $10 \mathrm{~cm}^{3}$, on reprend $9 \mathrm{~cm}^{3}$ (correspondant à $3 \mathrm{~cm}^{3}$ de lait) et on pratique la réaction des phénols comme ci-dessus.

\section{Exemple numérique}

Densité optique lue au Bonet-Maury (Ecran 15) $=68,0$ 5,2 (témoin) $=62,8$ correspond, d'après le grâphique de référence, à $0 \mathrm{mgr}$. 46 de tyrosine, d'où :

$\frac{0,46 \times 1.000}{3}=153$ milligrammes de tyrosine par litre de lait, ou : $153 \times 16,3=2$ gr. 49 de polypeptides par litre de lait, exprimé en caséine.

\section{Conclusions}

Nous avons donné, dans un précédent travail, un procédé colorimétrique basé sur le pouvoir chromogène des protides traité par le réactif des phénols, pour mesurer les protides totaux du lait.

Nous indiquons ici, comment on peut doser séparément la caséine, la lactalbumine et les polypeptides d'après ce même principe.

\section{BIBLIOGRAPHIE}

Folx et Denis. A colorimétric méthod for the détermination of phénol (and phénol dérivates) in urine. $J$. Biol. Chem., 1915, 22, 305.

R. Goiffon et Spaey. Mesure de l'Index. Tyrosine des polypeptides sériques. Bull. de la Soc. Chimie Biol., décembre 1934, p. 16.

Etienne Gorffon. Dosage pratique des protides du lait par la mesure colorimétrique de la tyrosine et du tryptophane. Le Lait, 1948, t. XXVIII, p. 449 .

\section{SÉCRÉTION LACTÉE HUMAINE ET ALIMENTATION HYDRIQUE (1)}

par

MaRCel LELONG, Mme F. ALISON et J. VINCENEUX

L'opinion qu'il est possible de modifier, la sécrétion lactée quantitativement et qualitativement par des variations quantitatives et qualitatives de l'alimentation est un préjugé encore fortement enraciné. E. Lambling recommandait d'ajouter, à la ration de la nourrice, un surplus de glucides et de lipides dans l'espoir

(1) Archives françaises de Pédiatrie, 1948, 5, no 4, 337. 